\title{
Stent-Assisted Coiling of a Ruptured Renal Artery Aneurysm: an Unusual Cause of Low Back Pain
}

\author{
Jorge A. Brenes* and Asad Irfanullah
}

Department of Medicine, Hennepin County Medical Center, Minneapolis, MN, USA

\begin{abstract}
Renal artery aneurysms are rare, but their reported incidence has increased as a result of the widespread use of imaging techniques. Most aneurysms are asymptomatic and discovered incidentally; however, they can present with refractory hypertension, pain and hematuria. We report the case of a 42 year old normotensive woman who presented with acute lower back pain with unremarkable lumbar x rays, in the setting of acute anemia with hypotension, leading to a high suspicion for internal bleeding. CT scan of the abdomen and pelvis revealed the presence of a ruptured $1.8 \mathrm{~cm}$ right renal artery aneurysm. Successful exclusion of the aneurysm was accomplished by stent-assisted coiling. Follow up imaging at one and six month intervals revealed patency of the stent and no deleterious effects on renal function.
\end{abstract}

Keywords: Back pain, renal artery, aneurysm, stent, coiling, Interventional Radiology.

\section{INTRODUCTION}

Acute low back pain is extremely frequent and represents the fifth most common reason for all physician visits [1]. Although it has a benign course in more than $90 \%$ of cases, serious conditions such as cancer, infection, fractures and visceral disease account for potentially lethal etiologies [2]. Within these, vascular catastrophies such as ruptured visceral aneurysms must be considered. Once thought to be extremely rare, renal artery aneurysms have been detected with increased frequency due to the widespread use of imaging techniques. Their risk of rupture is estimated to be as low as $0.17 \%$, but more importantly, mortality secondary to rupture can be as high as $80 \%$ [3]. The management of these lesions has evolved considerably over the last two decades with a variety of endovascular techniques available. We herein report the emergent percutaneous treatment of a spontaneously ruptured right renal artery aneurysm using stent-assisted coiling.

\section{CASE REPORT}

A 42 year old woman with history significant for GERD, constipation, external hemorrhoids, appendectomy, Csection and ventral hernia repair was transported to the emergency department (ED) two hours after the onset of acute right lower back pain following an attempt to bend down and pick up a heavy object. On arrival at the ED, she was afebrile with a blood pressure of $108 / 63 \mathrm{mmHg}$, pulse of $77 \mathrm{bpm}$, respiratory rate of 15 and oxygen saturation of $97 \%$ on room air. She was in severe pain despite receiving narcotics en route. Her physical examination was significant only for tenderness on palpation of the right paraspinous lumbar muscles. Straight leg testing was negative bilaterally and she had no neurological deficits. Lumbar spine x-rays did not show any abnormalities, urine pregnancy test was

*Address correspondence to this author at the Department of Medicine, Hennepin County Medical Center, Minneapolis, MN, USA;

Tel: 612-205 4158; Fax: 612-904 4226;

E-mail: jorgebrenes80@hotmail.com negative and her urinalysis was unremarkable. She was admitted to the General Medicine Service for further workup. Repeated physical examination revealed extension of the pain to her right flank and right upper quadrant, without signs of peritonitis or skin discoloration. Four hours after admission, the patient had a drop in her blood pressure to $87 / 43 \mathrm{mmHg}$, which partially responded to intravenous fluids. Her laboratory workup was remarkable for a creatinine of $1.2 \mathrm{mg} / \mathrm{dl}$, BUN of 18 , lactate of 2.4 $\mathrm{mmol} / \mathrm{L}$, WBC of 18.8 with $87 \%$ neutrophils and hemoglobin of $7.6 \mathrm{~g} / \mathrm{dl}$, down from $12.6 \mathrm{~g} / \mathrm{dl}$ two months prior, with an $\mathrm{MCV}$ of $88.9 \mathrm{fL}$. Emergent computed tomography (CT) scan of the abdomen and pelvis showed a moderate sized retroperitoneal hematoma caused by the rupture of a $1.8 \mathrm{~cm}$ saccular aneurysm arising from the right renal artery (Fig. 1). After evaluation by the on-call surgical team in conjunction with the interventional radiologist, the patient was deemed an acceptable candidate for endovascular therapy.

Informed consent was obtained from the patient. Access was gained into the right common femoral artery and a 5 French sheath (Cook Medical) was placed. A SOS selective catheter (Angiodynamics) was used to engage the right renal artery. Angiography demonstrated a large bilobed aneurysm arising approximately $25 \mathrm{~mm}$ distal to the origin of the renal artery (Fig. 2). The aneurysm was arising from the proximal aspect of a branch vessel supplying $30 \%$ of the posterior renal parenchyma. Over a Rosen wire (Cook Medical) a $45 \mathrm{~cm}$ long 7 French bright tip sheath (Cordis Medical) was advanced into the origin of the renal artery. A Renegade microcatheter (Boston Scientific) was then used to select the vessel exiting the aneurysm and $6 \mathrm{~mm}$ by $2 \mathrm{~mm}$ Tornado microcoils (Cook Medical) were deployed to favor stasis. Next the microcatheter was pulled back into the aneurysm and $8 \mathrm{~mm}$ by $4 \mathrm{~mm}$ coils were deployed within the aneurysm itself. Repeat angiogram demonstrated persistent filling of the mouth of the aneurysm. At this point it was felt that complete exclusion of the aneurysm with a covered stent was 


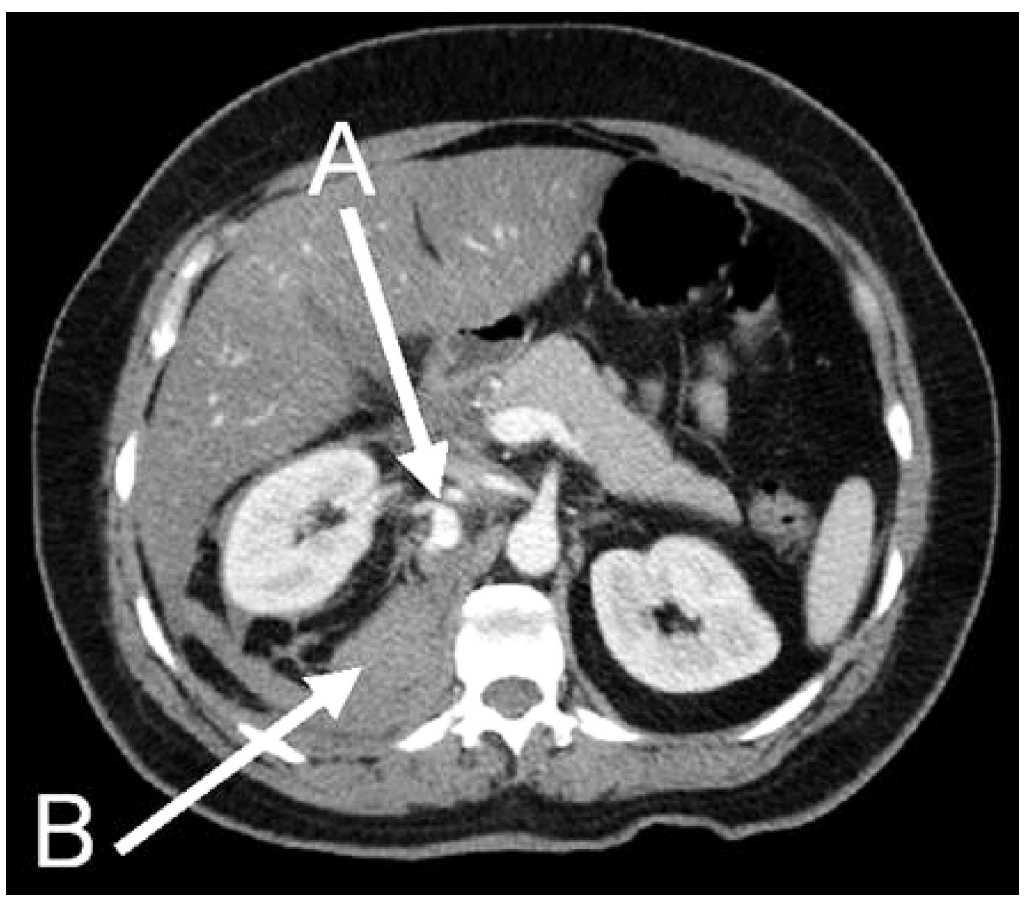

Fig. (1). Ruptured right renal artery aneurysm (A) with resulting moderate-sized retroperitoneal hematoma (B).

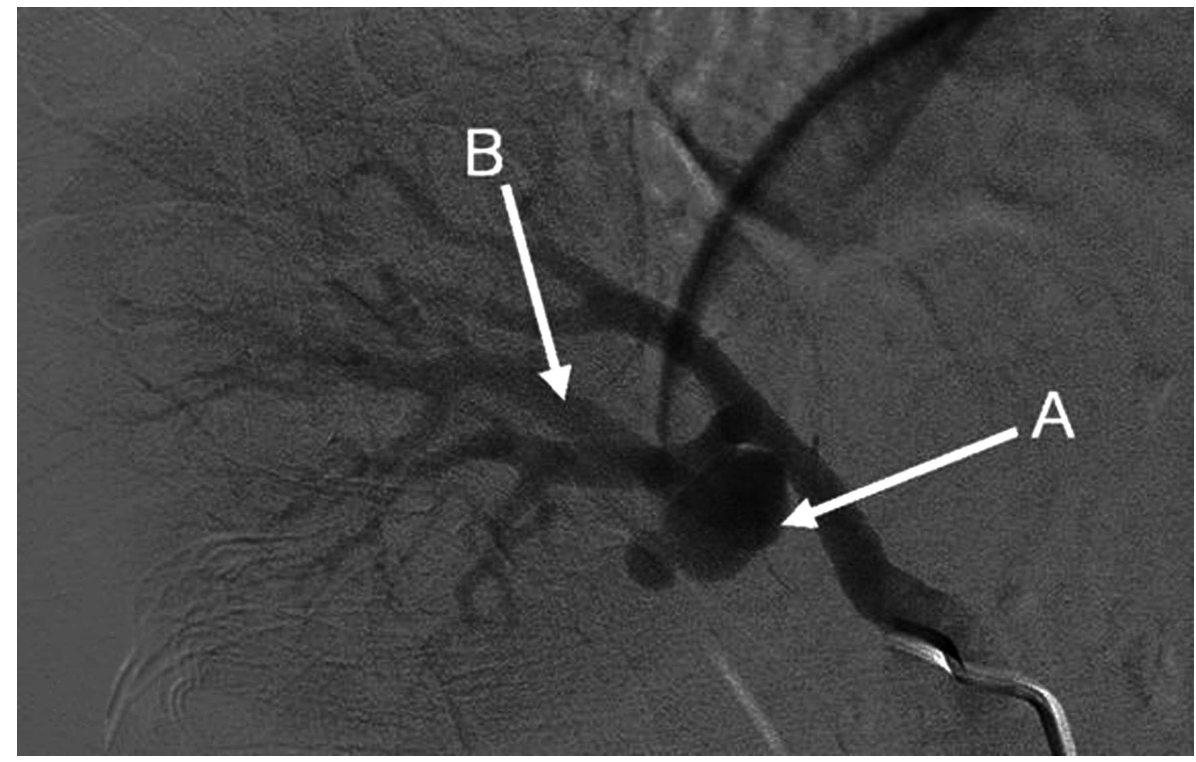

Fig. (2). Initial angiogram showing a small $1.8 \mathrm{~cm}$ bilobed, saccular renal artery aneurysm (A) proximal to a branch vessel (B) that perfused the posterior kidney.

the best option. A $6 \mathrm{~mm}$ diameter by $16 \mathrm{~mm}$ long Atrium ICAST stent (Atrium Medical) was then deployed within the distal main renal artery successfully covering the aneurysm. Final angiogram (Fig. 3) demonstrated complete exclusion of the aneurysm. Clinically, the patient's hemoglobin stabilized at $11.4 \mathrm{~g} / \mathrm{dl}$, her creatinine normalized to $0.6 \mathrm{mg} / \mathrm{dl}$ and she was discharged home on hospital day \# 5.

On postoperative day \#7, the patient again presented with flank pain and low grade fever. Repeat CT scan demonstrated a persistent, but stable, hematoma. $30 \%$ of the posterior renal parenchyma was infarcted secondary to the embolization of the branch vessel.Her presenting symptoms were likely secondary to post embolization syndrome.
Follow up CT scan at one month (Fig. 4) revealed adequate position of the stent and coils, with complete exclusion of the renal aneurysm; there was significant interval improvement of the retroperitoneal hematoma. Renal parameters, including a BUN of $14 \mathrm{mg} / \mathrm{dl}$ and a creatinine of $0.7 \mathrm{mg} / \mathrm{dl}$, remained stable. Repeat CT scan at six months (Fig. 5) confirmed patency of the stent with complete resolution of the retroperitoneal hematoma.

\section{DISCUSSION}

The first description of a renal artery aneurysm (RAA) is attributed to Rouppe in 1770 [4]. The true incidence in the general population is a matter of debate, ranging from $0.01 \%$ in autopsy studies to $0.7-1 \%$ in large scale screening studies 


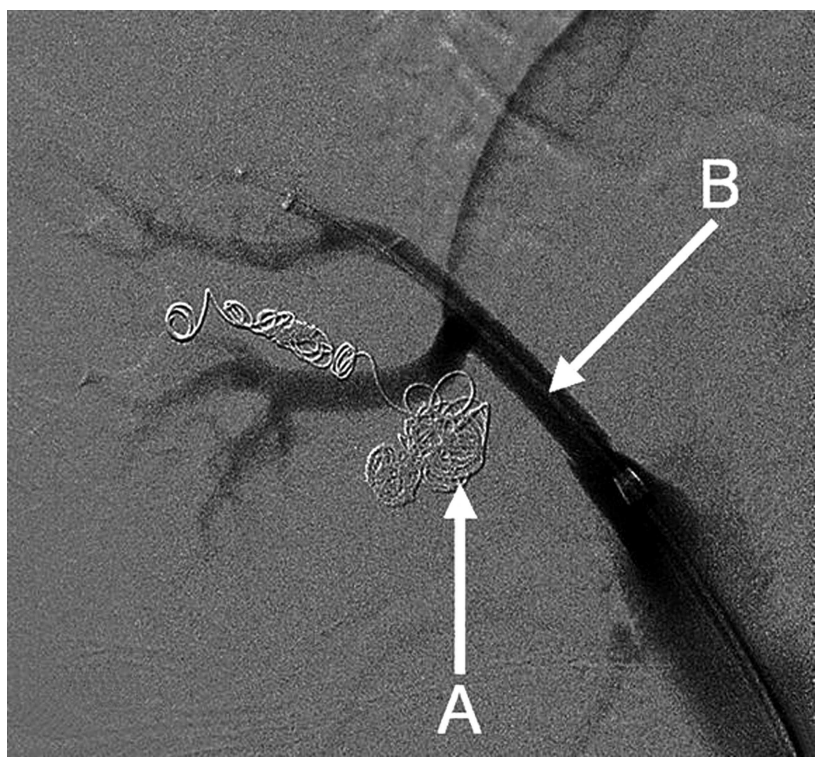

Fig. (3). Final angiogram which displays complete exclusion of the aneurysm (A) and posterior branch vessel with coils, and a stent (B) across the right main renal artery.

using imaging techniques [5]. RAAs are classified according to their primary or secondary etiology. Primary renal artery aneurysms are attributed to either arterial fibrodysplasia or atherosclerosis. Secondary renal artery aneurysms are due to a variety of causes such as pregnancy, trauma, iatrogenic, congenital malformations, malignancies, mycotic infections, neurofibromatosis, tuberous sclerosis, Ehlers-Danlos syndrome, and connective tissue disorders such as polyarteritis nodosa, Wegener's granulomatosis or Behcet's disease. Our patient did not have any significant cardiovascular risk factors and none of the other primary or secondary etiologies were identified.
More than $55 \%$ of renal artery aneurysms are asymptomatic and discovered incidentally during imaging procedures [5]. The most commonly associated sign is hypertension (73-89\%) and the most commonly associated symptoms are pain $(18-50 \%)$ and hematuria $(9-35 \%)[4,5]$. Our patient had a very unusual presentation, as she was normotensive, had no evidence of hematuria and the location of her pain was atypical according to most case reports. Pain arising from renal artery aneurysms is usually located in the flank or upper abdomen on initial presentation [6] and only few cases of low back pain have been described [7]. Immediate advanced imaging is rarely indicated in the initial evaluation of acute lower back pain according to the current guidelines [2]. Furthermore, a complete blood count is only indicated when there is a strong suspicion for malignancy or infection as the cause of back pain [1]. Vascular catastrophies, such as dissection or rupture of intraabdominal or retroperitoneal vessels can present with tachycardia, hypotension or sudden onset of protracted pain, which should prompt imaging. As stated previously, the most serious complication of renal artery aneurysm is rupture. Importantly, size alone has been shown to be an inaccurate predictor of rupture, as seen in our patient. [3]. Traditionally, a higher risk has been attributed to aneurysms of more than $20 \mathrm{~mm}$, however it can happen in lesions of less than $1 \mathrm{~cm}$ [9]. Most extraparenchymal renal artery aneurysms that bleed into the retroperitoneum are self contained, leading to better survival outcomes. In our patient, we believe that the development of a retroperitoneal hematoma created a protective tamponade effect which prevented further bleeding while she was taken to the Interventional Radiology suite.

Indications for the treatment of renal artery aneurysms should be individualized according to the patient's age, gender, blood pressure, renal function, aneurysm size and

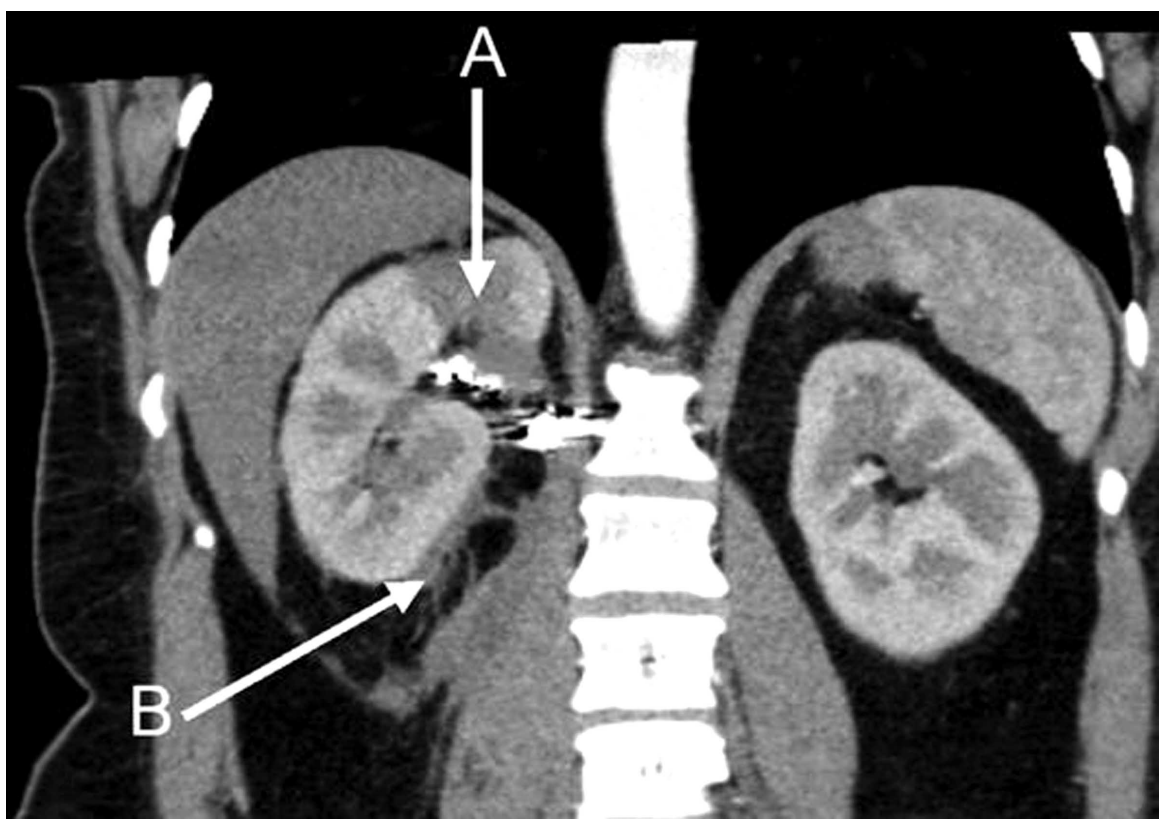

Fig. (4). CT scan at 1-month follow up shows residual infarction (A) from sacrifice of a posterior branch vessel and gradual resolution of retroperitoneal hematoma (B). 


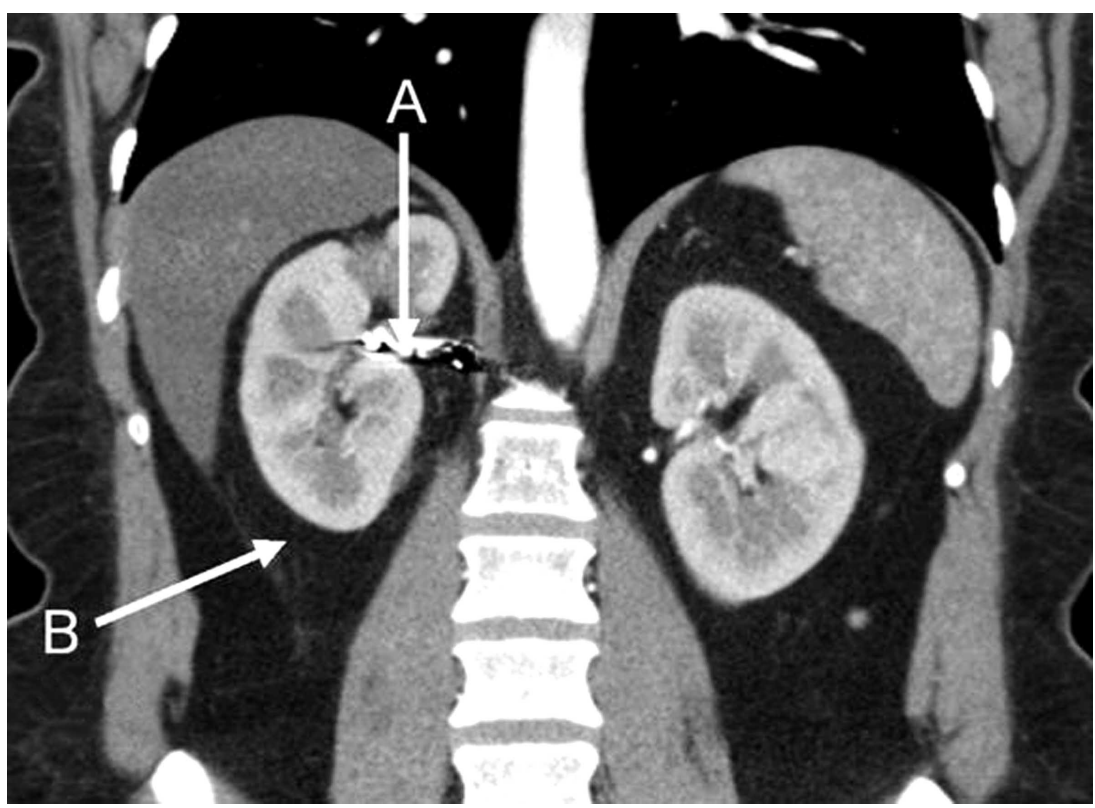

Fig. (5). CT scan at 6-month follow up exhibits patency of the stent (A) with complete resolution of the retroperitoneal hematoma.

complexity of treatment. Treatment is indicated for all renal artery aneurysms $\geq 2 \mathrm{~cm}$, most of those between $1.5-2 \mathrm{~cm}$ and those of at least $1 \mathrm{~cm}$ related to resistant hypertension. However, some groups [9] advocate for repair of all renal artery aneurysms regardless of their size in order to prevent microembolism and to avoid the development of fixed renal hypertension. Most authors recommend repair of all symptomatic lesions and any renal artery aneurysm in women of childbearing age [3].

Surgical and endovascular treatments share the common goal of preventing aneurysmal complications such as renovascular hypertension, renal artery thrombosis, infarction from distal embolization, arteriovenous fistula formation, expansion and rupture. Originally, almost all of these lesions were treated with nephrectomy. Recently, endovascular treatment of renal artery aneurysms has been in rapid evolution. Early on, it was limited to occlusive embolization of the feeding artery, but currently, a variety of options are available, including stent-graft exclusion of the aneurysm, simple coil embolization, stent assisted coiling and coil occlusion with sacrifice of the aneurysm parent artery. Selection of any given technique will depend on the particular anatomical features of the lesion (shape, neck size and specific location), technical feasibility and experience of the interventionist. Given the location of the aneurysm in this case the operator felt that the best endovascular option was a dual approach with coil embolization of the aneurysm and its branch vessel followed by placement of a stent. While a portion of normal renal parenchyma would be devascularized using this technique, given her normal renal function and a healthy contralateral kidney it was felt this would be of minimal clinical consequence to the patient. Case reports of endovascular repair for RAA show mortality rates close to $0 \%$ [8] but morbidity rates similar to those from surgery range from 8 to $18 \%$, often due to end organ ischemia, pain, fever, migration of embolizing materials or stent graft or complications in access site. As reported by other authors [8] and as seen in our case, sacrifice of branch vessels with concomitant loss of renal mass can be associated with significant patient discomfort and prolongation of hospital stay. However, these symptoms tend to be transient, with preservation of renal function and successful exclusion of the aneurysm on follow up.

Regardless of the chosen treatment, surveillance after the procedure is required to ensure that there is no reperfusion of the aneurysm from collateral flow or expansion due to continued exposure to arterial pressure [10]. Attention should focus on serial blood pressure measurements, as well as BUN and creatinine values. Non invasive imaging with color Doppler ultrasound or CT scan is recommended as well, but there are no consensus guidelines regarding the interval required. For stent related procedures, follow up studies will be needed, as their long term patency in the renal vasculature remains unknown.

\section{CONCLUSIONS}

Spontaneous rupture of a renal artery aneurysm is an unusual cause of acute back pain and expedited diagnostic testing is critical in view of its considerable morbidity and mortality. The need for nephrectomy is a rare event today as a variety of surgical reconstructive and minimally invasive techniques are available. Endovascular therapy has proven to be a feasible and effective approach for renal artery aneurysms, particularly in patients who are hemodynamically stable and have suitable anatomy. Further studies will be necessary to determine the long term outcome of this evolving percutaneous procedure as well as to establish guidelines regarding long term surveillance and follow up.

\section{CONFLICT OF INTEREST}

The authors declare that they have no conflict of interest.

\section{ACKNOWLEDGEMENTS}

The authors wish to thank Ed Peterson for his assistance with the figures, and Dr David $\mathrm{N}$ Williams for his kind review of the manuscript. 


\section{REFERENCES}

[1] Bach, S.M..; Holten, K.B. Guideline update: what's the best approach to acute low back pain? J. Fam. Pract., 2009, 58, 1-3.

[2] Kindade, S. Evaluation and treatment of acute low back pain. Am. Fam. Physician, 2007, 75, 1181-92.

[3] Eskandari, M.K.; Resnick, S.A. Aneurysms of the renal artery. Semin. Vasc. Surg., 2005, 18, 202-208.

[4] Kyle, V. Renal artery aneurysms. Can. Med. Assoc. J., 1968, 98, 815-818.

[5] Pride, Y.B.; Nguyen, M.C.; Garcia, L.A. Management of a renal artery aneurysm with coil embolization. J. Invasive Cardiol., 2008, 20, 470-472.

[6] Thomas, D. A case of ruptured aneurysm of the renal artery. ANZ. J. Surg., 1966, 36, 46-49.
Rundback, J.H.; Rizvi, A.; Rozenblit, G.N.; Poplausky, M.; Maddineni, S.; Crea, G.; Agrawal, U.; Olson, C.; Matalon, T.A.S. Percutaneous stent graft management of renal artery aneurysms. JVIR, 2000, 11, 1189-93.

[8] Tan, W.A; Chough, S.; Saito, J.; Wholey, M.H.; Eles,G. Covered stent for renal artery aneurysm. Catheter. Cardiovasc. Interv., 2001, 52, 106-109.

[9] Hupp, T.; Allenberg, J.R.; Post, K.; Roeren, T.; Meier, M.; Clorius, J.H. Renal artery aneurysm: surgical indications and results. Eur J Vasc Surg., 1992, 6, 477-486.

[10] Nosher, J.L.; Chung, J.; Brevetti, L.S.; Graham, A.M.; Siegel, R.L. Visceral and renal artery aneurysms: A pictorial essay on endovascular therapy. Radiographics, 2006, 26, 1687-1704.

Received: February 28, 2012

Revised: March 03, 2012

Accepted: March 03, 2012

(C) Brenes and Irfanullah; Licensee Bentham Open.

This is an open access article licensed under the terms of the Creative Commons Attribution Non-Commercial License (http://creativecommons.org/licenses/by-nc/3.0/) which permits unrestricted, non-commercial use, distribution and reproduction in any medium, provided the work is properly cited. 